\title{
JPEG Image Compression Using Fast 2-D DCT Technique
}

\author{
Sanjay J. Bagul ${ }^{1}$, Navinchandra G.Shimpi ${ }^{2}$, Pradeep M.Patil ${ }^{3}$ \\ Research Scholar, Electronics Engineering Department, North Maharashtra University \\ (N.M.U), Jalgaon, Maharashtra, India ${ }^{1}$ \\ Associate Professor, Chemical Engineering Department, North Maharashtra University \\ (N.M.U), Jalgaon, Maharashtra, India ${ }^{2}$ \\ Director and Principal, Electronics and Telecommunication Department, RMD Sinhgad Technical Institutes \\ Campus,Pune Maharashtra, India ${ }^{3}$
}

\begin{abstract}
Image Compression is a method, which reduces the amount of space required to store the image. The Discrete Cosine Transform (DCT) is a method that transforms a signal or image from spatial domain to frequency domain. This technique is widely used in image compression. In this paper, we present a lossless DCT compression technique for two-dimensional images. In several scenarios, the utilization of the presented technique for image compression results in comparable or better performance, when compared to the different modes of the lossless JPEG standard.
\end{abstract}

Keywords: Image Compression, JPEG, RLE, discrete cosine transform, 2-D DCT.

\section{INTRODUCTION}

Compression can be in the form of data compression, compression of images, audio data compression, video compression and bandwidth compression in
telecommunications. Techniques of image compression have been designed to manipulate the statistical redundancy present within images of real world. Emerging standards include MPEG for compression of motion video, JPEG for compression of still images and CCITT H.261 for compression of video telephony and teleconferencing. All these standards employ a basic technique known as the discrete cosine transform, which is developed by Ahmed et al in 1974 [1]. It is a lossless compression technique. The DCT is usually applied to reduce spatial redundancy in order to achieve good compression performance. Applications of two-dimensional DCT technique involve image compression and compression of individual video frames. DCT is useful for transferring multidimensional data from spatial domain to frequency domain, where different operations, such as spread spectrum, data compression, watermarking can be performed [2]. JPEG process is widely used form of lossy image compression that centers on the DCT. Fourier transforms and DCT convert images from spatial-domain to frequency-domain to de-correlate pixels [3]. JPEG is used for both color and black and white images. Two broad categories of compression are lossless and lossy compression. Lossless compression techniques never remove any information from the original image. These are referred to as bit preserving or reversible compression techniques [4]. Lossless compression frequently involves some form of entropy encoding based on information theory techniques. The algorithms for lossless compression are CCITT group 3 and 4 compression, flate/deflate compression, Huffman compression, LZW compression, RLE compression, Lossy compression creates smaller files by discarding excess image data from the original image. Audio and video compression techniques are most suited for this form of compression. JPEG compression is a lossy compression technique. Lossy compression methods are advantageous over lossless methods in some cases where lossy method produce a much smaller compressed file than any known lossless method.

\section{BASIC COMPRESSION METHODS}

A. JPEG compression: Joint Photographic Expert Group (JPEG) which is commonly used method of compression for photographic images. JPEG compression technique can be used in a variety of file formats like EPS-files, PDFfiles, etc. In this technique firstly the image is partitioned into non-overlapping $8 \times 8$ blocks. DCT is then applied to each block to convert the spatial domain gray level of pixels into coefficients in frequency domain. After computation of DCT coefficients, their normalization is performed according to a quantization table with different scales provided by the JPEG standard computed by psycho visual evidence. Quantized coefficients are rearranged in a zigzag scan order for further compression by an efficient lossless coding algorithm such as Huffman coding and run-length coding [5].

B. Run-Length Encoding (RLE): RLE stands for Run Length Encoding. This is a lossless algorithm that only furnishes decent compression ratios in specific type of data. It is a form of data compression in which the same data value occurs in many consecutive data elements (known as Runs) are stored as a single data value and count. This is useful on data that contains many such runs for example, simple graphic images like icons, line drawings, and animations. It may increase the file size as that does not have many runs and not useful with files. RLE compression can be used in the file formats like TIFF and PDF files. 
C. Huffman Coding: The Huffman compression algorithm is invented by David Huffman, formerly a professor at MIT. Huffman compression is a lossless compression algorithm that is apotheosis for compressing text or program files. This explains why it is used a lot in compression programs like ZIP or ARJ. Huffman encoding can be optimized in two different ways:

- Adaptive Huffman code dynamically changes the code words concordant to the change of probabilities of the symbols.

- Extended Huffman compression encodes groups of symbols rather than single symbols.

\section{III.PRESENTED METHODOLOGY USING DCT}

The image data is de-correlated by DCT. Each transform coefficient can be encoded without dropping off the compression efficiency, after de-correlation [6]. Two important forms of DCT are explained as follows:

One-Dimensional DCT: The DCT of a list of $\mathrm{n}$ real numbers $s(x)$, where $x=0,1_{v \ldots s} n-1$, is the list of length $n$ given by

$$
C(u)=\alpha(u) \sum_{x=0}^{N-1} f(x) \cos \left[\frac{\pi(2 x+1) u}{2 N}\right]
$$

: For $u=0,1_{s}, 2_{z}, N-1$

Similarly, the inverse transform is defined as

$f(x)=\sum_{x=0}^{N-1} C(u) \alpha(u) \cos \left[\frac{\pi(2 x+1) u}{2 N}\right]$

Thus, first transform coefficient is the coefficient having average value of the sample sequence.

Two-Dimensional DCT: The DCT is one of many transforms that takes its input and transforms it into a linear combination of weighted basis functions. The basis functions are commonly the frequency. 2-D Discrete Cosine Transform is a one dimensional DCT applied twice, once in $x$ direction and then in $y$ direction. One can imagine the computational complexity of doing so for large image.

$D(i, j)=\frac{1}{\sqrt{2 N}} C(i) C(j) \sum_{x=0}^{N-1} \sum_{y=0}^{N-1} \cos \left[\frac{(2 x+1) i \pi}{2 N}\right] \cos \left[\frac{(2 y+1) j \pi}{2 N}\right]$

where,

$C(u)= \begin{cases}\frac{1}{\sqrt{2}}, & \text { if } u=0 \\ 1, & \text { if } u>0\end{cases}$

$N$ is the size of the block that the DCT is applied on. The equation calculates one entry $\left(i, j^{\text {th }}\right)$ of the transformed image from the pixel values of the original image matrix. For standard $8 \times 8$ block used in JPEG compression, $N$ equals $\mathrm{X}$ and $x$ and $y$ range from 0 to 7 . Thus, $D\left(i_{x} j\right)$ is given by equation:

$D(i, j)=-\frac{1}{4}(i) C(j) \sum_{x=0}^{7} \sum_{y=0}^{7} p(x, y) \cos \left[\frac{(2 x+1) i \pi}{16}\right] \cos \left[\frac{(2 y+1) j \pi}{16}\right]$

As DCT uses cosine functions, resulting matrix depends on the horizontal and vertical frequencies. Thus, an image block with a lot of change in has a very random looking resulting matrix of a large value for the first element and zeroes for the other element.
The process of image compression using DCT may be described as follows:

Step 1 The image first is broken into $8 \times 8$ blocks of pixels.

Step 2 The DCT is applied to each block, working from left to right and top to bottom.

Step 3 Each block is compressed using quantization table.

Step 4 The array of compressed blocks that comprise the image is stored in a drastically reduced amount of space.

The reconstruction of image is performed through decompression which is a process that uses the Inverse Discrete Cosine Transform (IDCT).

\section{EXPERIMENTAL RESULT}

The evaluation of performance of the presented scheme is done by applying 2-D DCT on Lena's image $352 \times 352$ as a test image. Initially, DCT is applied to rows only through compression factor 2,4 and 8 and observe the result. DCT is then applied to row $\mathrm{x}$ column. The results are then compared with various compression methods. We used Peak Signal-to Noise Ratio (PSNR) and Mean Square Error (MSE) for a compressed image. This ratio is used as quality measurement between the original and compressed image.
1) Image of size $352 \times 352$ :
a) DCT to rows only

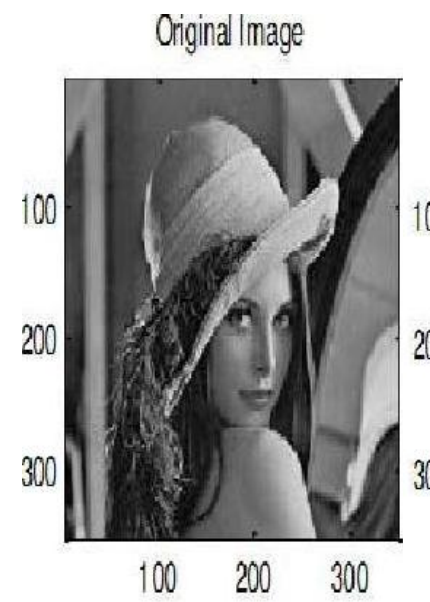

Compression Factor 2

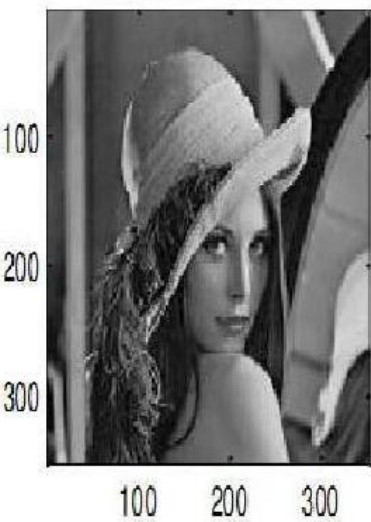

Compression Factor 4

Compression Factor 8

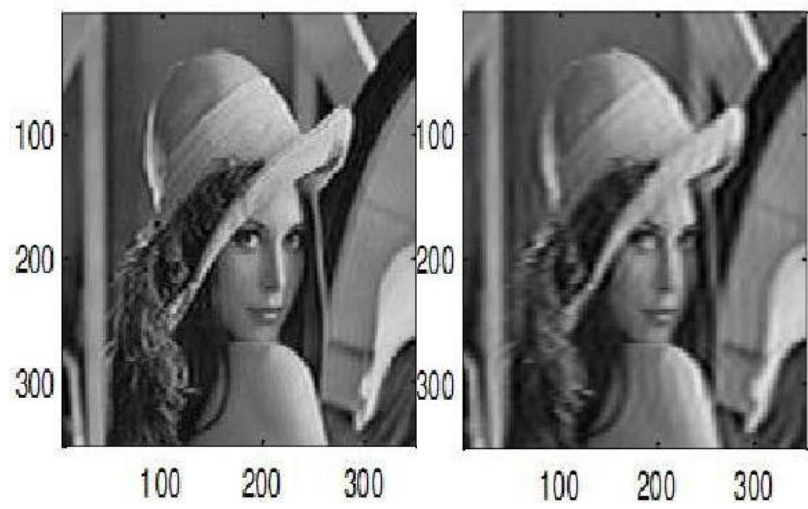


b) DCT to rows $\times$ columns:

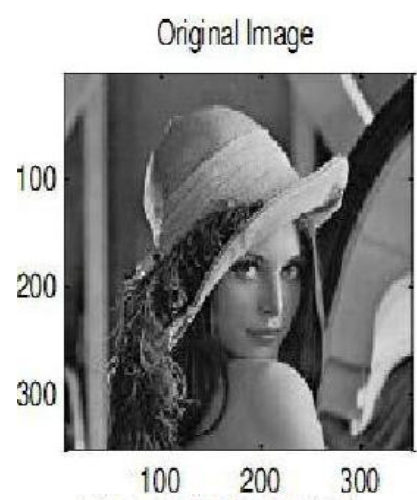

Compression Factor 4 " 4
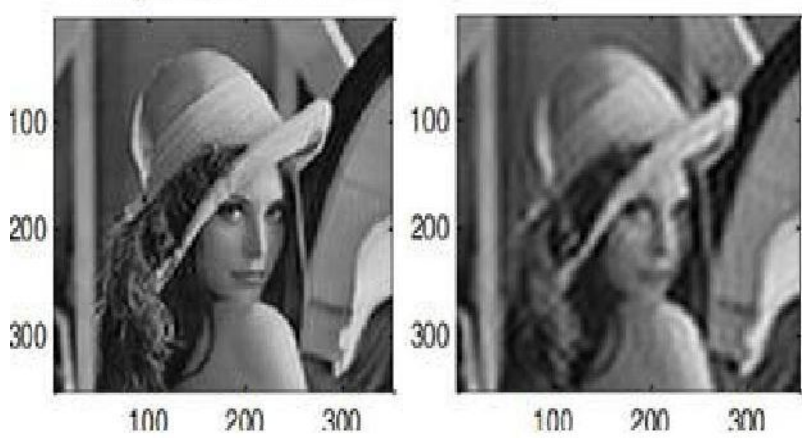

\section{CONCLUSIONS}

In this paper, there are 256 possible shades of gray in a black and white picture, and a difference of say 10 is hardly obtrusive to the human eye. DCT takes the advantage of redundancies of data by grouping pixels with similar frequencies. Thus, the difference between original and de-compressed image goes on decreasing as there is an increase in image resolution at the same compression ratio. This image compression scheme is presented based on the 2-D DCT. The anticipating results obtained relevant reconstructed image quality as well as preservation of significant image details, while accomplishing high compression rates.

High compression ratio and better image quality accomplished which is better than existing methods. The focus of this paper was on the development of efficient and effective algorithm for still image compression. Algorithm for fast and lossless compression using 2-D DCT is developed. The compression results show reduction in encoding time with little degradation in image quality compare to subsisting method. The compression ratio is also increased compared to other methods. The future work involves improving image quality by increasing PSNR value and lowering MSE value.

\section{REFERENCES}

[1] N. Ahmed, T. Natarajan and K.R. Rao, "Discrete Cosine Transform," IEEE Transactions on Computers, vol. C-23, no. 1, pp. 90-93, Jan 1974.

[2] A. B. Watson, "Image Compression Using the Discrete Cosine Transform," Mathematica Journal, vol. 4, no. 1, pp. 81-88, 1994.

[3] N. R. Thota and S. K. Devireddy, "Image Compression Using Discrete Cosine Transform ," Georgian Electronic Scientific Journal: Computer Science and Telecommunications, vol. 3, no. 17, pp. 35-43, 2008.
[4] Anshuman, G Jaiswal and A Rai, "Image Compression Using Discrete Cosine Transform And Wavelet Based Transform," National Institute of Technology, Rourkela, B. Tech Thesis 2007.

[5] G. K. Wallace, "The JPEG still picture compression standard," Communications of ACM -Digital Multimedia Systems, vol. 34, no. 4, pp. 30-44, Apr 1991.

[6] E. Feig and S. Winograd, "Fast Algorithms for the Discrete Cosine Transform," IEEE Transactions on Signal Processing, vol. 40, no. 9, pp. 2174-2193, Sept 1992.

\section{BIOGRAPHIES}

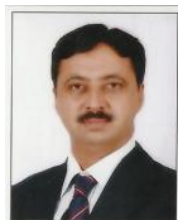

Mr. Sanjay J. Bagul received his B. E. (Electronics) degree in 1994 from University of Pune, Pune, (India) and M.E. (Instrumentation) degree in 1999 from Shri Ramanand Terth Marathwada University (SRTMU), Nanded, (India). He worked as Lecturer and Assistant Professor in department of Electronics Engineering at various engineering colleges in North Maharashtra University, Jalgaon and Pune University, (India). Presently he is working as Principal, NIT's Late Annasaheb patil Polytechnic, Panchavati, Nashik, (India). He is member of ISTE. He has been recognized as a P.G. Teacher by University of Pune in the state of Maharashtra (India). He has been published paper in various international and national journals and conferences.

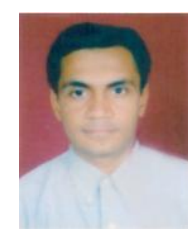

Dr. Navinchandra Gopal Shimpi completed his $\mathrm{Ph}$. $\mathrm{D}$ in Polymer from North Maharashtra University, Jalgaon MS India. Presently, he is working as a Head \& Assistant Professor in the Department of Nanoscience and Technology, University Institute of Chemical Technology, North Maharashtra University, Jalgaon. He has published more than forty research papers in International and National journal of good impact factor. Besides this, he has presented more than forty papers in international and thirty research papers in national conferences. He has filled three Indian patents and one is granted. Dr. Navinchandra Shimpi has organized nine conferences and staff development program in the capacity of Convener and Coordinator. His area of research is synthesis and characterization of nanomaterials, conducting polymers, biodegradable polymers, rubber nanocomposites, carbon nanotubes etc. In his supervision thirteen students

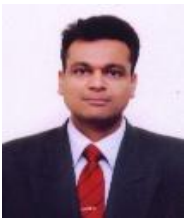

Dr. Pradeep Mitharam Patil received his B. E. (Electronics) degree in 1988 from Amravati University, Amravati, (India) and M. E. (Electronics) degree in 1992 from Marathwada University, Aurangabad, (India). He received his Ph.D. degree in Electronics and Computer Engineering in 2004 at Swami Ramanand Teerth Marathwada University, (India). From 1988 to 2011 he worked as Lecturer and Assistant Professor and Professor in department of Electronics Engineering at various engineering colleges in Pune University, (India). Presently he is working as Dean, RMD Sinhgad School of Engineering and Director of RMD Sinhgad Technical Institutes Campus, Warje, Pune, (India). He is member of various professional bodies like IE, ISTE, IEEE and Fellow of IETE. He has been recognized as a $\mathrm{PhD}$ guide by various Universities in the state of Maharashtra (India). His research areas include pattern recognition, neural networks, fuzzy neural networks and power electronics. His work has been published in various international and national journals and conferences including IEEE and Elsevier. 